\title{
Implementation of a Web-based NASA World Wind Tool for the Visualization of Climate Data
}

\author{
Sandro ARCIDIACONO \\ Carinthia University of Applied Sciences, Villach/Austria · sandro.arcidiacono@edu.fh-kaernten.ac.at
}

This contribution was double-blind reviewed as extended abstract.

\begin{abstract}
This paper outlines the implementation of a web-based tool for the visualization of climate data in the area of Villach using the Java based virtual globe framework World Wind of the National Aeronautics and Space Administration (NASA). The used climate data is provided by the research project Sensors4All, developed by the Carinthia University of Applied Sciences (CUAS), School of Geoinformation and Environmental Technologies due to the departments research focus on "Environmental Monitoring" by accomplishing projects concerning Sensor Web Enablement (ANDRAE et al. 2009, ANDRAE \& SIMONIS 2011) and sensor data validation (ANDERS \& HECKE 2011). The Sensor4All sensor network itself is currently limited to 15 sensors that are situated on the roofs of the CUAS sites in Villach and Feldkirchen, on top of the mountain Dobratsch and on roofs of other well-situated educational institutions in the area of Villach. Since May 2011 they are continuously collecting data of the weather phenomena temperature, precipitation, humidity, air pressure and wind speed (HECKE et al. 2012).

The only way of viewing the collected data of the Sensors4All research project in a cartographic manner was to send a request to a GeoServer that returned an interpolated Web Mapping Service (WMS) data layer of the last temperature measurement (DE LA BEAUJARDIERE 2006). This layer could be included in Google Earth to get an idea of the affected area and the temperature profile. Due to the fact that a user could neither view any past measurements, nor choose another phenomenon, the visualization tool was developed. It enables the user not only to decide between different phenomena in a specific time frame, but also to change the height gradient for temperature, precipitation and air pressure, view climate diagrams and exclude measurements of specific sensors from visualization. Additionally the visualized layer can be aggregated by using the maximal, minimal, average or summation of the measured values of the chosen sensors in the specific time frame. The possibility to load external WMS layers was also implemented, so that the previous visualization method of Sensors4All and a certain degree of expandability is provided.
\end{abstract}

\section{Problem Definition}

As already partially described in the Abstract, the main problem is that no proper cartographic visualization of the collected data had been implemented in the Sensors4All system. The only two approaches that were implemented are the use of a GeoServer to publish 
the last temperature measurements for all sensors and a Web Client where a user has the possibility to see Charts of measurements in a specific time span. Therefore a proper visualization tool had to be implemented that has the benefit that a user has the possibility to select different phenomena and sensors to be visualized in a specific time frame. The NASA World Wind framework was chosen due to its high grade of mutability. This results from the idea of this framework, where many examples and a specific basis are given, but most of the implementation is left to the programmer.

\subsection{System Architecture}

The already existing Sensors4All environment is used for acquisition, storing and transmission of the climate data. Every 15 minutes the measured climate data is sent from the sensors to the PostGIS database. The stored data is published via a Sensor Observation Service (SOS) server that is receiving data of the deployed sensors. This server receives requests from a so called SOS-Facade, a Java servlet running on a Tomcat webserver. A Facade is a software design pattern that is implemented in the servlet. It provides a simplified interface for the SOS-Server and helps connecting it to another component, an ArcGIS-Python script. The script is getting temperature data of the last measurement from all sensors over the SOS-Facade and is interpolating it for the GeoServer. Then the script restarts the GeoServer for actualizing the specific layer on it. A Google Earth client is then able to request the interpolated temperature layer via the GeoServers WMS interface. The Sensors4All system that is used as a basis for the visualization tool is shown as darker components in figure 1 . To reach the expected results, a new component had to be added to the already existing system. This component is the visualization tool, a java application, based on the NASA World Wind framework, which is communicating on one hand with the GeoServer or any other free selectable WMS and on the other hand with the SOS-Facade servlet on the webserver. The communication with the servlet is necessary to retrieve sensor information and sensor data needed for further calculations and functions, like the interpolation and visualization of the climate data. The connection to the GeoServer can be established manually in the tool for requesting the former temperature data as WMS layer. The appended components are shown as brighter components in figure 1.

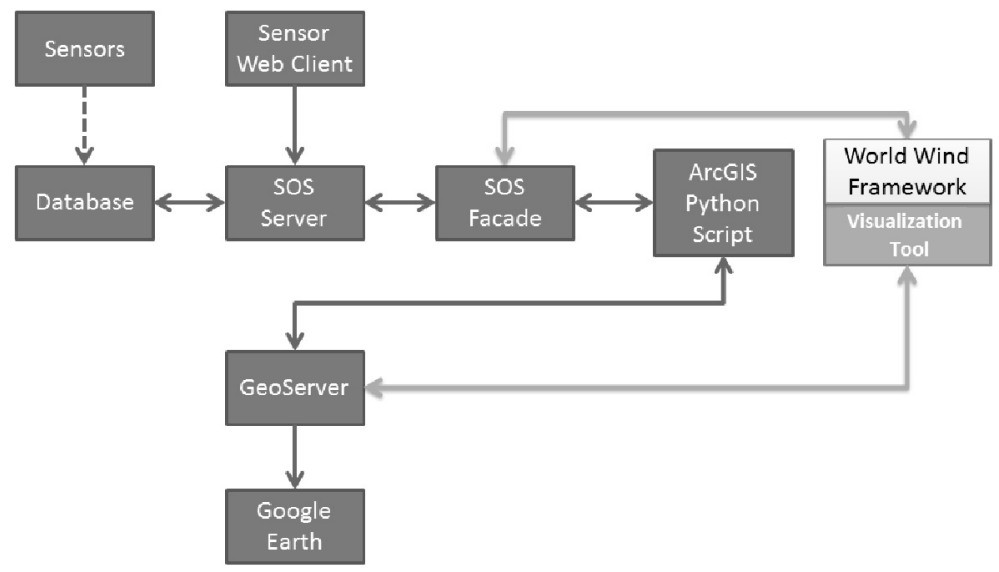

Fig. 1: System diagram of the Sensors4All system in dark gray and the new visualization tool modules in light gray 
The visualization tool itself requests the data from the SOS Facade via an HTTP request and gets an Extensible Markup Language (XML) structure as response. It includes all information about the selected sensors, like the coordinates and the measurement values of the chosen phenomenon in the selected period of time.

\section{Results}

In figure 2 the overall result of the visualization tool can be seen. In the upper left corner a menu is situated where all features can be found. Below a layer list is shown, where each layer can be checked or unchecked to control its visibility on the map. In the lower left corner a control for switching the 3D globe into a flat projected 2D surface can be seen. The virtual globes map is implemented with a compass in the upper right and an overview map in the upper left corner. Different other control features are situated at the bottom of the map window to ensure a simplified navigation.

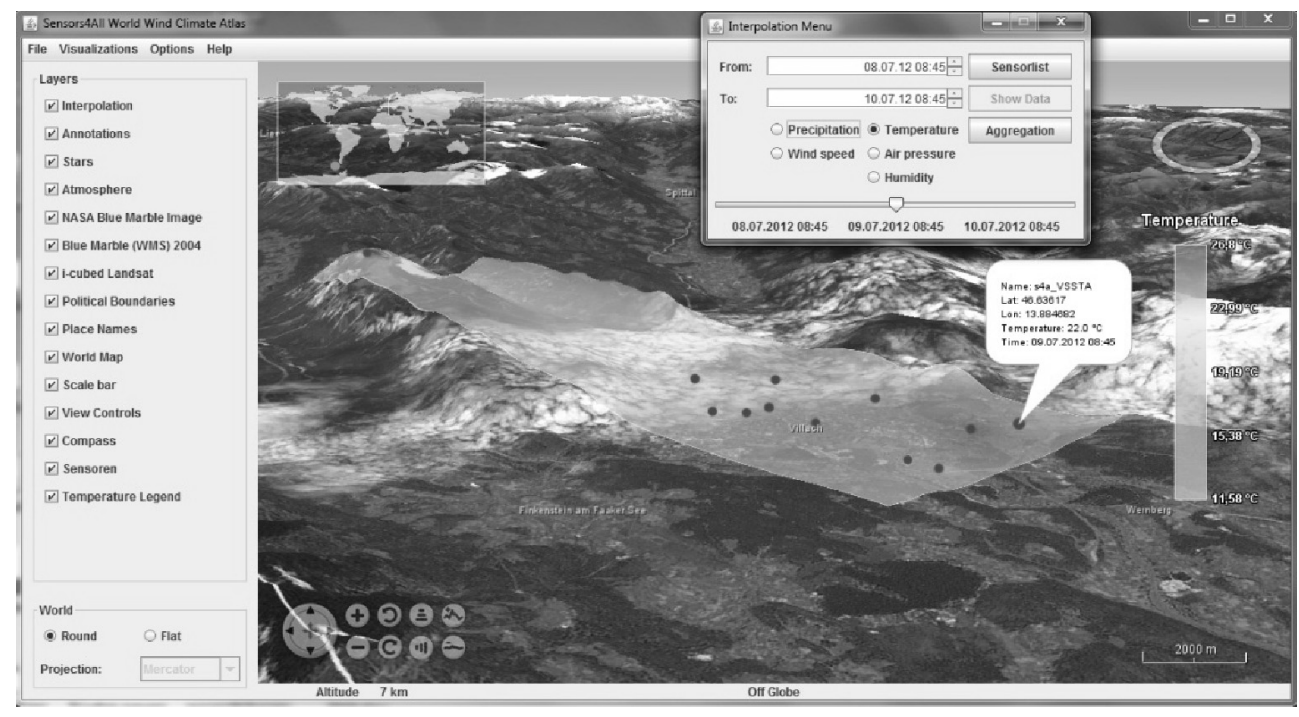

Fig. 2: Overall result of the Visualization tool with the options in the interpolation menu, a sensor annotation and the sensor visualization with the temperature phenomenon

In the map window the result of the interpolation over a period of time is shown. For the sensor visualization pink and orange colored points that lye on height of the surface at the specific position were chosen, so that the sensors could easily be highlighted from the rest of the map. An orange point means that the specific sensor was not chosen for the actual interpolation, whereas the measurement values of the pink points have been included in the actual calculations. As interpolation method Inverse Distance Weighting was chosen due to the fact that it is more appropriate for a small amount of location points (SLUITER 2009, LU \& WONG 2008). The slider that is situated in the interpolation menu enables the user to 
select a specific time point within the chosen period of time. By dragging the slider, the chosen sensors are interpolated at the selected time shown between the start- and end-time below the slider. The annotations of the affected sensors can be seen by moving the mouse to the specific sensor, showing the measured value of the selected time point and phenomenon. Optionally the height gradients of the phenomena temperature, precipitation and air pressure can be changed or disabled. This feature changes the calculated values of the interpolated surface, so that a relation between the height and the phenomenon can be seen on the map. Additionally a climate diagram for each sensor in the selected time frame can be shown to the user to visualize the change of temperature and precipitation over the chosen time interval. For that the open source Java framework JFreeChart was used (JFREECHART 2011). The climate diagram itself has three axes - two y axes and one $\mathrm{x}$ axis. The left $\mathrm{y}$ axis shows the precipitation in millimetres and the right y axis shows the temperature in ${ }^{\circ} \mathrm{C}$. The $\mathrm{x}$ axis on the bottom states the time frame. Different legends have been implemented, showing an appropriate color visualization of the chosen phenomenon and its given measurement values in the chosen time frame. As seen in figure 2 the temperature phenomenon is chosen for this example. Therefore the legend spans from the lowest to the highest measured temperature, using a color spectrum that spans from dark blue $\left(-25^{\circ} \mathrm{C}\right)$ to dark red $\left(+50{ }^{\circ} \mathrm{C}\right)$. Also for the phenomena humidity, air pressure and wind speed the full color spectrum from dark blue to dark red is used. Precipitation has an own legend that uses the colors from light blue (low-precipitation) to dark blue (heavy precipitation).

\section{References}

ANDERS, K. \& HeCKE, A. (2011), Data Quality Web-based service for Sensor Networks. In: Conference Journal for the International Cartographic Conference 2011, Paris, France.

Andrae, S., Gruber, G., Hecke A. \& Wieser, A. (2009), Sensor Web Enablement Standards and Open Source Implementations for Observation Data. In: Conference Journal for the International Snow Science Workshop 2009, Davos, Switzerland.

AndRAE, S. \& SimONIS, S. (2011), OpenSensors: A community platform to enable the Sensor Web and Foster Earth Observation Research. In: Proceedings of the IST Africa 2011, Botswana.

De La Beaujadiere, J. (2006), OpenGIS® Web Map Server Implementation Specification. In: Open Geospatial Consortium Inc. (Ed..), OpenGIS ${ }^{\circledR}$ Implementation Specification. United States of America.

Hecke, A., Anders, K., Tomintz, M., Wieser, A. \& Gruber, G. (2012), Sensors4All Sensor Networks for Pupils Based on OGC Standards. In: Conference Proceedings for GI_Forum 2012: Geovisualization, Society and Learning 2012, Salzburg, Austria.

JFREEC $\bar{C}$ HART (2011), JFreeChart, free open-source chart library. http://www.jfree.org/jfreechart/ (last visited: 08.01.2013).

LU, Y. G. \& WONG, W. D. (2008), An adaptive inverse-distance weighting spatial interpolation technique, Computers \& Geosciences, Vol. 34, No. 9, 1044-1055.

SLUITER, R. (2009), Interpolation methods for climate Data - Literature Review. KNMI intern rapport, Royal Netherlands Meteorological Institute, De Bilt. 